\title{
Do Quantum Objects Have Temporal Parts?
}

\author{
Thomas Pashby
}

10th January, 2013.

1. Introduction This paper provides a new context for an established metaphysical debate regarding the problem of persistence. Namely, how can it be said that one and the same physical object persists through time while changing over time? I contend that a popular view about persistence which maintains that objects persist by perduring - that is, by having temporal parts—receives a particularly neat formulation in quantum mechanics due to the existence of a formal analogy between time and space. I argue, however, that on closer inspection this analogy fails due to a 'no-go' result which demonstrates that quantum systems can't be said to have temporal parts in the same way that they have spatial parts. Therefore, if quantum mechanics describes persisting physical objects, then those objects cannot be said to perdure.

This argument serves two aims. The first is to continue the recent tradition of addressing the problem of persistence in the context of specific physical theories: Balashov (2010); Gilmore (2008) consider special relativity; Butterfield (2005, 2006) considers classical mechanics. The second aim is to provide a novel interpretation of the no-go result mentioned above, which is well-known in the quantum foundations literature but rarely discussed by philosophers of physics. The result is often phrased like this: There exists no time observable 
canonically conjugate to the Hamiltonian. This fact was first observed by Pauli in 1933, and there are various proofs which arrive at essentially this conclusion. In contrast to Halvorson (2010) I regard this result not as an argument against the existence of time but rather as an argument that quantum objects (described by pure states) cannot have temporal parts.

I begin in Section 2 by introducing popular views of persistence and suggest my own classification scheme that includes room for another view of persistence which I term temporal holism. In Section 3 I examine some details of the quantum mechanical formalism and suggest how to apply these views of persistence to realist ontology of persisting quantum objects. I propose that a perduring quantum object has temporal parts in the same way as it has spatial parts. In Section 4 I motivate and present a suitable account of the spatial parts of a quantum object, which leads in Section 5 to a concrete suggestion for how such an object could be said to have temporal parts. However, this suggestion is ruled out by the no-go result mentioned above. I conclude by surveying some alternative accounts of temporal parts and possible implications for the persistence debate.

2. The Metaphysics of Persisting Objects The debate over the question of how physical objects persist has garnered much attention in recent years, and has come to be regarded as a question of metaphysics in its own right, roughly orthogonal to debates in the metaphysics of time. While the contemporary debate began as a straight fight between endurantism (the view that persisting objects are individuals without temporal extension, wholly present at every time they exist) and perdurantism (the view that persisting objects are temporally extended individuals that persist by having temporal parts), following Sider's (1997) intervention another combatant—stage theory — was added to the mix. According to stage theory, 
persisting objects are concatenations of appropriately related ${ }^{1}$ but distinct instantaneous stages and thus it disagrees with both endurantism (which says that a persisting object is the same individual at every instant) and perdurantism (which says that a persisting object is not composed of many individual stages but is an individual having temporal parts).

These views of persistence can thus be classified according to how they divide a persisting object up into individuals, ${ }^{2}$ and as to whether persisting objects can be said to have temporal parts. Endurantism and perdurantism have in common the view that a persisting object is a single individual: for both the endurantist and perdurantist, 'the table at time $t$ ' picks out the same individual as 'the table at time $t$ ' (where $t \neq t$ ' are instants in the lifetime of the table). However, the perdurantist considers 'the table' to be a four-dimensional entity that extends through time and space, whereas the endurandist maintains that 'the table' is an entity with only spatial extent. While the endurantist believes the (same) table is wholly present at every time it exists, the perdurantist believes that only part of the table is wholly present at any one time: the perdurantist believes that ordinary objects like tables are temporally extended and have temporal parts.

Like perdurantism, stage theory maintains that persisting objects have temporal parts. As a result they are sometimes referred to as varieties of a single view: four-dimensionalism. However, according to stage theory 'the table at time $t$ ' refers to a particular instantaneous stage rather than part of a temporally extended individual, and 'the table at time $t$ " refers to a different instantaneous stage. For the stage theorist, a persisting object comprises a collection

\footnotetext{
${ }^{1}$ Hawley (2004) suggests that this is a transtemporal counterpart relation that resembles David Lewis' transworld counterpart relation.

${ }^{2}$ My use of the word 'individual' is intended to be interpretation neutral, serving to avoid prejudicial use of the contentious term 'persisting object.'
} 
of these instantaneous stages, each of which has the attribute being a table, and none of which has temporal extension. So the stage theorist believes that a persisting object like a table is in fact composed of many individuals_-momentarily existing table stages. While stage theory and perdurantism agree that persisting objects have temporal extension, according to stage theory the temporal parts of a persisting object are not mereological parts of a four-dimensional entity but rather instantaneous stages (or collections of such stages).

Note that these three views are thus completely differentiated by their answers to two questions: (a) Is a persisting object a single individual? (b) Does a persisting object have temporal parts? There is, therefore, a forth view possible which answers yes to the first but no to the second. I call this view temporal holism, the view that persisting objects are temporally extended but mereologically simple individuals. ${ }^{3}$ I will argue that quantum mechanics makes trouble for the perdurantist's notion of a temporal part, which suggests that temporal holism is compelled upon one who wishes to hang on to the idea that a persisting 'quantum object' is a single temporally extended individual. The next section provides an account of quantum objects, and how they may be said to persist.

\footnotetext{
${ }^{3}$ This discussion closely relates to the classification of Gilmore (2008). Question (b) corresponds to his mereological perdurance / endurance distinction, while question (a) relates to his locational perdurance / endurance distinction. However, the latter distinction relies on the predicate 'exactly occupies' whose application to unitarily evolving quantum systems is problematic (for reasons I discuss in Section 4). Regardless, I take temporal holism to capture something of his idea of "a singly located and temporally extended but mereologically simple electron" (1229).
} 
3. Persisting Objects in Quantum Mechanics Quantum mechanics provides our best theory of matter, and its empirical predictions have been startlingly accurate. That much is uncontroversial. On the other hand, any attempt to assert exactly why it has proved so successful, or precisely what it tells us about the nature of material objects involves taking sides on disputes regarding its interpretation that have lasted over 80 years. To avoid taking sides, I will proceed by specifying under what conditions one would be committed to regarding the quantum state as describing a persisting material object. Nonetheless, I take it that prima facie a realist metaphysician who takes tables (composed of collections of complex organic molecules) to be persisting objects would be compelled to similarly regard, say, a molecule of Buckminsterfullerene $\left(C_{60}\right)$ composed of sixty atoms of carbon, which has been seen to display distinctly quantum behavior.

First, some details about the formalism of ordinary (non-relativistic) quantum mechanics. As our concern is with spatio-temporal properties, we will consider systems with no internal degrees of freedom (i.e. spinless particles). Therefore, the state space of the theory is provided by the space of square integrable functions defined over all of space, that is, infinite-dimensional (separable) complex Hilbert space $\mathcal{H}=L^{2}\left(\mathbb{R}^{3}\right)$. The pure states $|\psi\rangle$ are in one-to-one correspondence with the one-dimensional subspaces of $\mathcal{H}$ or, equivalently, the set of independent unit vectors which individually span those subspaces. Since $\mathcal{H}$ is a vector space, linear combinations of pure states are also pure states (this is the superposition principle). In what follows I will only consider pure states.

The first interpretative posit I require is realism, the claim that real physical systems are authentically described by quantum mechanical states. The next posit I require is completeness, the claim that a pure state provides a complete description of an individual quantum system which leaves nothing out (i.e. no hidden variables). So far we would be 
justified in claiming that the quantum state describes a physical object. But what about persisting objects? For that we require some facts about quantum dynamics, which takes two forms: the Schrödinger and Heisenberg pictures.

In the Schrödinger picture, the history of a system is given by a family of states $|\psi(t)\rangle$, parameterized by $t \in \mathbb{R}$. Once the state of the system at a particular time is given, the entire family is determined according to the time-dependent Schrödinger equation in terms of a one-parameter (strongly continuous) group of unitary operators $U_{t}=e^{-i H t}$, where $H$ is the Hamiltonian of the system. If a pure state $|\psi(0)\rangle$ describes a physical object which exists at time $t=0$, then the states $|\psi(t)\rangle$ describe the lifetime of a persisting object which exists at each time $t$ in the state $|\psi(t)\rangle$. The infamous measurement problem arises when we consider the relation of the unitary dynamics of the state to the results of laboratory observations. To avoid having to address this issue, I will consider only systems undergoing unitary evolution, which corresponds to the assumption that quantum objects are isolated.

In the Heisenberg picture, the observables change with time rather than the state of the system. The time dependence of a (Schrödinger picture) observable $A$ (a self-adjoint operator on $\mathcal{H}$ ) is again given in terms of the unitary group $U_{t}$. The corresponding Heisenberg picture observable is $A(t)=U_{t}^{\dagger} A U_{t}$ and in the Heisenberg picture the state of the system at all times is $|\psi\rangle$. If $|\psi(t)\rangle=U_{t}|\psi\rangle$ then the two pictures are empirically equivalent, returning the same expectation values $\langle A\rangle=\left\langle\psi_{t}|A| \psi_{t}\right\rangle=\left\langle\psi\left|U_{t}^{\dagger} A U_{t}\right| \psi\right\rangle=\langle\psi|A(t)| \psi\rangle$. However, they are not necessarily equivalent with respect to metaphysics: the states $|\psi(t)\rangle$ appear suited to describe individuals existing at a single instant whereas $|\psi\rangle$ appears to describe an individual with temporal extension, existing at many times.

Interpreted in this way, the difference between the Schrödinger and Heisenberg pictures corresponds precisely to the distinction I drew between (respectively) endurantism and stage 
theory on the one hand, and perdurantism and temporal holism on the other. According to an endurantist interpretation, the Schrödinger picture states $|\psi(t)\rangle$ are distinct states of a single individual whose identity through time transcends the assignment of differing properties at different times. Arguably this is the interpretation which best fits the common understanding of time evolution as describing the changing state of a system whose identity through time is assumed. This is consistent with endurantism's claim to provide an account of our intuitive grasp of persistence.

However, if we take the view instead that each state $|\psi(t)\rangle$ corresponds to a distinct quantum object then it appears that the Schrödinger picture family of states describes a series of distinct instantaneously existing quantum objects related by the unitary group $U_{t} \cdot{ }^{4}$ This closely resembles stage theory. If the instantaneous stages correspond to the Schrödinger picture states $|\psi(t)\rangle$, then the temporal parts of a persisting quantum object correspond to sets of those states i.e. according to stage theory the temporal part extending from $t_{1}$ to $t_{2}$ is the collection of instantaneous stages described by the set of states $\left\{|\psi(t)\rangle: t \in\left[t_{1}, t_{2}\right]\right\}$.

In contrast, the Heisenberg picture describes a single quantum object $|\psi\rangle$ which exists at many times. According to perdurantism, $|\psi\rangle$ is an object which persists by having temporal parts. Temporal holism would amount to the denial of the perdurantist's claim that persisting objects have temporal parts. For this debate to make sense, we require a notion of what it is for $|\psi\rangle$ to have temporal parts. In order to find one, I return to the motivation for the claim that persisting objects have temporal parts, which is a strong analogy between the way an object extends in time and space—-between temporal parts and spatial parts.

Perdurance corresponds to the way a road persists through space; part of it is here and part of it is there, and no part is wholly present at two different places. (Lewis

\footnotetext{
${ }^{4}$ More precisely, they will be distinct if $U_{t}$ is non-periodic.
} 
1986, 202)

As I see it, the heart of four-dimensionalism is the claim that the part-whole relation behaves with respect to time analogously to how it behaves with respect to space. (Sider 1997, 204)

Thus a suitable account of what is is for a quantum object $|\psi\rangle$ to have temporal parts will closely resemble a satisfactory account of how a quantum object may be said to have spatial parts. In the following section I provide just such an account.

4. Parts and Spatial Parts It is well known that there are severe difficulties in regarding the wavefunction $\psi(x)$ as describing 'the way that a quantum object persists through space.' This is because $\psi(x)$ (the position representation of the vector $|\psi\rangle$ ) is not a function of three-dimensional space but of $3 N$-dimensional configuration space, so the $x$ in the argument only corresponds to physical space in the special case of a single particle. This problem is not insurmountable, however, since there is nonetheless a way of describing $|\psi\rangle$ as a mereological sum of component quantum objects, each of which is confined to a particular region of space.

In order to do so, we require a suitable part-whole relation. I contend that such a relation may be found by considering the subspaces of $\mathcal{H}$, or equivalently the projections onto those subspaces. According to classical mereology, the relation of parthood is (minimally) reflexive (everything is part of itself), transitive (if $p$ is part of $q$ and $q$ is part of $r$ then $p$ is part of $r$ ) and antisymmetric (no two distinct things can be part of each other). As is well known, the subspaces of a vector space labeled $A, B, C \ldots$ are partially ordered by the relation of inclusion, which is reflexive ( $A \subseteq A$ ), transitive (if $A \subseteq B$ and $B \subseteq C$ then $A \subseteq C$ ) and antisymmetric (if $A \subseteq B$ and $B \subseteq A$ then $A=B$ ). 
I claim that in quantum theory the spatial parts of a quantum object $|\psi\rangle$ may be given in terms of the subspaces of $\mathcal{H}$ associated with the spectral decomposition of the position observable $Q$. The reason this provides a suitable decomposition into spatial parts is that $Q$ thus serves to uniquely associate every region of space $\Delta$ with a projection operator $P_{\Delta}$ on $\mathcal{H}$ such that disjoint regions of space are assigned to mutually orthogonal subspaces. ${ }^{5}$ By projecting a vector state $|\psi\rangle$ onto the subspace associated with $\Delta$ one obtains a state $P_{\Delta}|\psi\rangle=\left|\psi_{\Delta}\right\rangle$ which may be interpreted as describing a quantum object wholly located within $\Delta$, related to $|\psi\rangle$ as part of a whole. I now proceed to fill in some details of this account.

Quantum mechanical observables such as position are associated with self-adjoint operators (position in three-dimensional space is associated with three operators $Q_{x}, Q_{y}, Q_{z}$ ). The set of self-adjoint operators on $\mathcal{H}$ is in one-to-one correspondence with the set of Projection Valued Measures (PVMs) on $\mathcal{B}(\mathbb{R})$, the Borel subsets of $\mathbb{R} .{ }^{6}$ Such a PVM is an assignment of projections on $\mathcal{H}$ to the measurable subsets $\Delta \subset \mathcal{B}(\mathbb{R})$ such that the map $\Delta \mapsto P_{\Delta}$ has the properties: 1. $P_{\mathbb{R}}=\mathbb{I}$ (normalization), and 2. $P_{\cup_{n} \Delta_{n}}=\sum_{n} P_{\Delta_{n}}$ ( $\sigma$-additivity) (where $\Delta_{n} \subset \mathcal{B}(\mathbb{R})$ is a sequence of mutually disjoint subsets and convergence is in the weak operator topology).

The association of projections with spatial regions $\Delta$ through the position observable satisfies the quantum mechanical parthood relation I articulated above in a way that makes it quite plausible that they provide a suitable notion of spatial parts. Due to 2. the relation of

${ }^{5}$ For a general proof that this is always the case in non-relativistic quantum mechanics see (Wightman 1962).

${ }^{6}$ This is in effect a statement of the spectral theorem. See, for example, (Teschl 2009, Thm. 3.7). 
subspace inclusion provided by the $P_{\Delta}$ corresponds precisely to the corresponding relation of regions of space. For example, if $\Delta^{\prime}$ is a region of space that lies within $\Delta$ then $P_{\Delta^{\prime}}$ projects onto a subspace that lies within the subspace that $P_{\Delta}$ projects onto, and, since the $P_{\Delta}$ are projections, 2. also entails that disjoint regions of space are associated with mutually orthogonal subspaces.

$P_{\Delta}$ is commonly known as a localization system, in the sense that performing a measurement of a projection $P_{\Delta}$ has the possible outcomes $\{0,1\}$ : either the system is located in $\Delta$ or the system is not located in $\Delta$, with probabilities supplied by the Born rule. These possibilities are mutually exclusive in that $P_{\mathbb{R} / \Delta}=\mathbb{I}-P_{\Delta}$ (2.) and so a system may be said to be 'wholly located in $\Delta$ at $t$ ' on the condition that $P_{\Delta}\left|\psi_{\Delta}(t)\right\rangle=\left|\psi_{\Delta}(t)\right\rangle$, in which case we say that $\left|\psi_{\Delta}(t)\right\rangle$ is an eigenstate of $P_{\Delta}$ at time $t$. Since in general the system will not be in an eigenstate of any projection $P_{\Delta}$ we can't say that it located anywhere in particular but rather that it is localizable.

Another characteristic of the PVM $P_{\Delta}$ which justifies the contention that it provides an assignment of spatial parts is that this assignment covaries with spatial translations $U_{a}^{\dagger} P_{\Delta} U_{a}=P_{\Delta-a}$, where $U_{a}=e^{-i P a}$ is the one-parameter unitary group of spatial translations in the direction of $a$ generated by the (total) momentum $P$. Roughly, this is a consequence of the fact that $Q$ and $P$ are canonically conjugate, $[Q, P]=i \hbar$. Viewing these transformations passively, as moving the origin of the spatial co-ordinates by $a, P_{\Delta-a}|\psi\rangle$ in the new co-ordinates denotes the same part as $P_{\Delta}|\psi\rangle=\left|\psi_{\Delta}\right\rangle$ in the old. Viewed actively, covariance assures us that the part $U_{a}\left|\psi_{\Delta}\right\rangle$ is just the part $\left|\psi_{\Delta}\right\rangle$ moved to a new location $\Delta+a$ since $P_{\Delta+a} U_{a}\left|\psi_{\Delta}\right\rangle=U_{a}\left|\psi_{\Delta}\right\rangle$.

According to my definition, then, the spatial part of $|\psi(t)\rangle$ located in $\Delta$ is the eigenstate of $P_{\Delta}$ obtained by projection of $|\psi\rangle$ i.e. given by the state $P_{\Delta}|\psi(t)\rangle$. These spatial parts are thus 
defined instantaneously: at each instant $P_{\Delta}$ supplies an assignment of parts to spatial regions. But note that this discussion has taken place in the Schrödinger picture. In the Heisenberg picture the projections $P_{\Delta}(t)$ are time indexed and we have $P_{\Delta}(t)\left|\psi_{\Delta}\right\rangle=U_{t}^{\dagger} P_{\Delta} U_{t}\left|\psi_{\Delta}\right\rangle=\left|\psi_{\Delta}\right\rangle$. Thus the Heisenberg picture projections pick out subspaces that are invariant under time translations, whereas the Schrödinger picture subspaces covary with time translations. This aspect might be thought of as a boon for the perdurantist since it (apparently) restores a symmetry between temporal and spatial parts by defining spatial parts directly in terms of spatial regions (Butterfield 1985). However, I argue in the next section that quantum mechanics instead breaks this symmetry by failing to allow an analogous notion of temporal parts.

5. (No) Temporal Parts I proposed that the spatial parts of a quantum object $|\psi\rangle$ are the projections onto the subspaces associated with spatial regions by a localization scheme $P_{\Delta}$. The states $\left|\psi_{\Delta}\right\rangle=P_{\Delta}|\psi\rangle$ deserve to be regarded as spatial parts since $\left|\psi_{\Delta}\right\rangle$ describes a quantum object wholly located in region $\Delta$ which bears the parthood relation to $|\psi\rangle$. By analogy, then, the temporal part wholly present during the time interval $I=\left[t_{1}, t_{2}\right]$ would be a quantum object $P_{I}\left|\psi_{I}\right\rangle$ which can be interpreted as being present only at times $t \in\left[t_{1}, t_{2}\right]$, and which bears the relation of parthood to $|\psi\rangle$.

The spatial parts $\left|\psi_{\Delta}\right\rangle$ may be regarded as wholly located in $\Delta$ since they are eigenstates of $P_{\Delta}$, a PVM that associates regions of space with subspaces of $\mathcal{H}$. Thus the temporal parts of $|\psi\rangle$ would be quantum objects $\left|\psi_{I}\right\rangle$ that are eigenstates of $P_{I}$, a projection associated with the interval $I$, with all the $P_{I}$ together forming a PVM. This requirement ensures that the subspaces associated with disjoint intervals of time are mutually orthogonal so that $\left|\psi_{I}\right\rangle$ may be interpreted as an object present during $I$ but at no other times. 
The interpretative difficulty with such a state $\left|\psi_{I}\right\rangle$ is that, since $P_{J}\left|\psi_{I}\right\rangle=0$ for disjoint $I, J$, it appears be a state that violates probability conservation (if this is interpreted literally as saying that the system does not exist at other times). However, just as the existence the PVM $P_{\Delta}$ (the spectral decomposition associated with position) does not imply the existence of quantum mechanical systems that persist in an eigenstate of some $P_{\Delta}$, the existence of $P_{I}$ (the spectral decomposition associated with time) need not imply that any systems actually exist in such states. Specifically, just as the existence of $P_{\Delta}$ is equivalent to the existence of a 'position basis' (a resolution of the identity) in which a vector state may be written $\langle x \mid \psi\rangle$, the existence of $P_{I}$ is equivalent to the existence of a 'time basis' in which the same vector may be written $\langle t \mid \psi\rangle$.

However, there is a further requirement that one should demand in order to view $P_{I}$ as providing an assignment of temporal parts: as the spatial parts $\left|\psi_{\Delta}\right\rangle$ were stable under spatial translations, so too should the temporal parts $\left|\psi_{I}\right\rangle$ be stable under temporal translations. Thus $P_{I}$ needs to covary with time shifts so that $U_{t}^{\dagger} P_{I} U_{t}=P_{I-t}$. The reasons for doing so are as before: the definition of a temporal part should not depend on a particular co-ordinatization of the time axis, and a temporal part wholly present during $I$ when shifted in time by $t$ should be identical with the part wholly present during $I+t$.

Unfortunately for the would-be quantum perdurantist, it turns out that these two requirements are in conflict with the restriction on physical Hamiltonians known as the spectral condition, which permits only Hamiltonian operators with a spectrum bounded from below i.e. only systems whose energy cannot decrease without bound. The usual argument for this requirement is that to do otherwise would allow for systems which may transfer energy to their surroundings indefinitely. While it is true that all systems we know obey the spectral condition (e.g. a free particle or harmonic oscillator), we could also view it as a principle of 
the theory on par with (say) the first law of thermodynamics.

Now, it is a theorem that if (i) $P_{I}$ is a PVM, (ii) $U_{t}^{\dagger} P_{I} U_{t}=P_{I-t}$, and (iii) $U_{t}$ is a unitary group generated by a self-adjoint operator $H$ whose spectrum is bounded from below, then $P_{I}=0$ for all $I .^{7}$ This result is often referred to as Pauli's theorem, after a argument that appeared in a footnote of Pauli's handbook article of 1933 which sought to establish that there can be no self-adjoint operator $T$ canonically conjugate to the Hamiltonian $H$. One can see by the aforementioned correspondence of PVMs with self-adjoint operators that this theorem has that implication, although there is some ambiguity in the phrase 'canonically conjugate'. 8

I have argued that we should view requirements (i) and (ii) as necessary for the states $P_{I}|\psi\rangle$ to be temporal parts of $|\psi\rangle$. Since this theorem establishes that $P_{I}|\psi\rangle=0$ (the zero vector) for all $I$ and all $|\psi\rangle$, it serves as a reductio of the perdurantist's claim that $|\psi\rangle$ persists through time by having temporal parts in the same way as it persists through space by having spatial parts. In other words, no quantum object has temporal parts.

How might the perdurantist respond? One could attempt to hijack the stage theorists definition of a temporal part as the set of states $\left\{\left|\psi_{t}\right\rangle: t \in\left[t_{1}, t_{2}\right]\right\}$ by defining projections as integrals over one-dimensional projectors onto those states,

$$
P_{I}^{\tau}=\int_{t_{1}}^{t_{2}} d t|\psi(t)\rangle\langle\psi(t)| .
$$

These projections covary since $U_{t}^{\dagger} P_{I-t} U_{t}=P_{I}^{\tau}$ but they do not project onto orthogonal states. But in the absence of an appropriate parthood relation it is not clear that the state $P_{I}^{\tau}|\psi\rangle$ that results can be regarded as a temporal part of $|\psi\rangle$. Furthermore, this state combines the Heisenberg picture and Schrodinger picture in an uncomfortable way. While with appropriate

${ }^{7}$ See Srinivas and Vijayalakshmi (1981, Theorem 1) or Halvorson (2010).

${ }^{8}$ See Galapon (2002) for a critique of Pauli's argument along these lines. 
normalization the various projectors $P_{I}^{\tau}$ may sum to unity, the complex amplitudes $\langle\psi(t) \mid \psi\rangle$ that result depend directly on the value of $t$ since by definition $|\psi\rangle=|\psi(0)\rangle .{ }^{9}$

Another way to relax condition (i) while holding onto (ii) involves the use of Positive Operator Valued Measures (POVMs) rather than PVMs. While a time PVM assigns a projection operator $P_{I}$ to a temporal interval $I$, a time POVM would provide an assignment of positive operators $E_{I} \geq 0$ to temporal intervals $I$ which nonetheless obey conditions 1 . and 2 . of the previous section. While the quantum objects $E_{I}|\psi\rangle$ thus behave somewhat like temporal parts without being assigned to orthogonal subspaces, the interpretation of these parts as being wholly present during $I$ is problematic. It seems to me that rather than providing an account of temporal parts consistent with perdurantism, this is better understood as an articulation of temporal holism i.e. as an account of how an assignment of properties to times can be consistent with the denial that there exist temporal parts.

6. Conclusion This attempt to transpose the traditional debate regarding the metaphysics of persistence into the formalism of quantum mechanics has had some interesting outcomes. First, it was suggested that there is another possible view of persistence which has received little attention: temporal holism, the view that persisting objects are four-dimensional individuals without temporal parts. Second, I provided an account of what it would be for a quantum object to have spatial parts, and argued for an analogous account of temporal parts. Finally I showed that this account, although attractive, is actually ruled out by quantum

\footnotetext{
${ }^{9} \mathrm{~A}$ more promising alternative for the perduranist involves regarding the true home of a persisting quantum object not as $\mathcal{H}$ but rather the direct sum of temporally indexed Hilbert spaces $\mathcal{H}_{t}$, each spanned by the complete set of instantaneous Schrödinger picture states $\{|\psi(t)\rangle:|\psi\rangle \in \mathcal{H}\}$, with the projectors $P_{I}^{\tau}$ acting on this 'larger' Hilbert space.
} 
mechanics and therefore persisting quantum objects do not have temporal parts, and so cannot be said to perdure.

To the extent that we have reason to think that all persisting objects are quantum objects, this provides reason to doubt that perdurantism is true. ${ }^{10}$ Since the application of stage theory and endurantism to persisting objects in quantum mechanics was relatively unproblematic, this confers some support to these views instead. However, I have not considered here relativistic quantum mechanics, which introduces problems for the Schrödinger picture on which these views rely. ${ }^{11}$ Reasons to discount the Schrödinger picture are thus reasons to discount stage theory and endurantism, and so if relativistic quantum mechanics were to compel the adoption of the Heisenberg picture for independent reasons then it seems that temporal holism would win out as the last view standing. This would be a surprising victory for a view of persistence that has received almost no attention in the metaphysics literature. Detailed consideration of the complications introduced by relativity (including the problem of making contact with the existing relativistic persistence debate) will, however, have to await further investigation.

\footnotetext{
${ }^{10}$ Arguably, classical persisting objects are best thought of as "patterns that emerge from an ubiquitous, continuous, and very efficient process of decoherence.” Butterfield $(2006,41)$. Decoherence refers to the process by which interactions between an 'object' system (e.g. a dust particle) and its environment serve to pick out a dynamically 'preferred' basis according to which the object system is approximately diagonalized. My argument concerns the basis independent description of the entire system of object and environment.

${ }^{11}$ In particular, Rovelli (2004) advocates the Heisenberg picture as providing a relativistically invariant notion of the quantum state, a view that he traces back to Dirac.
} 


\section{References}

Balashov, Y. (2010). Persistence and spacetime. Oxford: Oxford University Press.

Butterfield, J. (1985). Spatial and temporal parts. The Philosophical Quarterly 35(138), $32-44$.

Butterfield, J. (2005). On the persistence of particles. Foundations of Physics 35(2), 233-269.

Butterfield, J. (2006). The rotating discs argument defeated. The British Journal for the Philosophy of Science 57(1), 1-45.

Galapon, E. (2002). Pauli's theorem and quantum canonical pairs. Proceedings of the Royal Society of London A 458(2018), 451-472.

Gilmore, G. (2008). Persistence and location in relativistic spacetime. Philosophy Compass 3(6), 1224-1254.

Halvorson, H. (2010). Does quantum theory kill time? http://www.princeton.edu/ hhalvors/papers/notime.pdf.

Hawley, K. (2004). How Things Persist. Oxford: Clarendon Press.

Lewis, D. K. (1986). On the Plurality of Worlds. Oxford: Blackwell.

Rovelli, C. (2004). Quantum Gravity. Cambridge: Cambridge University Press.

Sider, T. (1997). Four-dimensionalism. The Philosophical Review 106(2), 197-231.

Srinivas, M. and R. Vijayalakshmi (1981). The 'time of occurrence' in quantum mechanics. Pramana 16(3), 173-199. 
Teschl, G. (2009). Mathematical Methods in Quantum Mechanics. Providence: American Mathematical Society.

Wightman, A. S. (1962). On the localizability of quantum mechanical systems. Reviews of Modern Physics 34, 845-872. 